Bangladesh J. Bot. 41(1): 71-85, 2012 (June)

\title{
FLORISTIC DIVERSITY OF DHAMRAI UPAZILA OF DHAKA WITH EMPHASIS ON MEDICINAL PLANTS
}

\author{
M Oliur Rahman*, Rumana Tanjin Antara, Momtaz Begum and Md Abul Hassan
}

Department of Botany, University of Dhaka, Dhaka 1000, Bangladesh

Key words: Angiosperm diversity, Dhamrai, Medicinal plants, Bangladesh

\begin{abstract}
A total of 263 angiosperm species under 210 genera and 79 families have been recorded from Dhamrai Upazila of Dhaka district. Of these, Magnoliopsida is represented by 200 species under 154 genera and 62 families while Liliopsida is represented by 63 species under 56 genera and 17 families. Asteraceae is the largest family in Magnoliopsida represented by 17 species, and Poaceae is the largest family in Liliopsida represented by 20 species. Habit analysis shows that herbs, shrubs and trees are represented by 166, 23 and 74 species, respectively. Sixty two medicinal plants have been documented with their uses for the cure of more than 30 diseases, and some of these are diabetes, jaundice, diarrhoea, dysentery, spleen and liver complaints, chronic ulcers, bronchitis, rheumatism, irregular menstruation, piles, urinary problems and heart diseases. Threats to the species have also been assessed and appropriate conservation measures suggested.
\end{abstract}

\section{Introduction}

Dhamrai is one of the five Upazilas of Dhaka district with an area of $307.4 \mathrm{~km}^{2}$ and is situated about $38 \mathrm{~km}$ north-west of Dhaka metropolis. It is located at $23^{\circ} 55^{\prime} \mathrm{N}$ and $90^{\circ} 14^{\prime} \mathrm{E}$. Dhamrai represents an undulating area with conspicuous crests and troughs. The soil is a heterogenous assortment of dry, moist and clay material. The crest soils are represented by three different types, namely brownish grey fine sandy loam, dark grey fine sandy loam and grey fine sandy loam. The trough soils are represented by three different types, i.e. greyish-yellow fine sandy loam, yellowish-grey fine sandy loam and grey sandy loam. The structure of soil of Dhamrai is medium angular to subangular, blocky and granular. Consistency of dry soil is hard, moist soil is slightly firm to friable, while nature of wet soil is slightly sticky or plastic. Soil pH ranges from $6.1-6.4$ (Hossain et al. 2003). Dhamrai Upazila enjoys the same climatic condition as other parts of the district do in the tropical monsoon zone. The temperature of the area ranges from $14.8-35.6^{\circ} \mathrm{C}$. Monthly humidity varies from $48-79 \%$ throughout the year. The highest humidity is noted in August and the lowest in February (BBS 2009).

The plants of Dhamrai area exhibit a diverse habitats, such as wetland, cultivated land, char, homestead area, scrub jungles, fallow lands, etc. which support luxuriant formation of angiosperms and play a vital role in the local economy, environment and primary health care system. The importance of studying local floristic diversity has been realized and carried out in Bangladesh by Khan et al. (1994), Rahman and Hassan (1995), Uddin and Rahman (1999), Khan and Huq (2001), Uddin and Hassan (2010). Recently Tutul et al. (2009, 2010) and Rahman et al. (2010) conducted the inventorying of angiosperm diversity of Runctia Sal forest of Bangladesh. The present study aimed at making an inventory of the angiosperm species of Dhamrai Upazila and to document the medicinal uses of plant species by local people.

*Author for correspondence: <dr_oliur@yahoo.com> 


\section{Materials and Methods}

Plant specimens have been collected from all over Dhamrai through several field trips covering all seasons during 2009 - 2010. The collected specimens have been critically examined, studied and identified at Dhaka University Salar Khan Herbarium (DUSH). Identifications have been confirmed by consulting standard literature (Khan 1972 - 1987, Khan and Rahman 1989 2002, Dassanayake and Fosberg 1980 - 1985), and matching with identified herbarium specimens housed at DUSH and Bangladesh National Herbarium (DACB). Nomenclature has been updated following recent literature including Ahmed et al. (2007 - 2009) and Rashid and Rahman (2011). The families are arranged (Table 1) according to Cronquist (1981), and the species are listed alphabetically under each family along with their Bangla name, habit, phenology and voucher number. Emphasis has also been given to the economically important species particularly the medicinal plants used as primary health-care. The information on the uses of medicinal plants has been gathered through interview of the local people. The voucher specimens are preserved at DUSH.

\section{Results and Discussion}

The present study has revealed a total of 263 angiosperm species in 210 genera under 79 families recorded from Dhamrai Upazila. Magnoliopsida is be represented by 62 families, 154 genera and 200 species, while Liliopsida is represented by 17 families, 56 genera and 63 species. In Magnoliopsida, Asteraceae appears to be the largest family having 16 genera and 17 species, followed by Fabaceae with 13 species and Euphorbiaceae with 10 species. In Liliopsida, Poaceae appears to be the largest family with 19 genera and 20 species followed by Araceae with 9 species and Cyperaceae with 7 species.

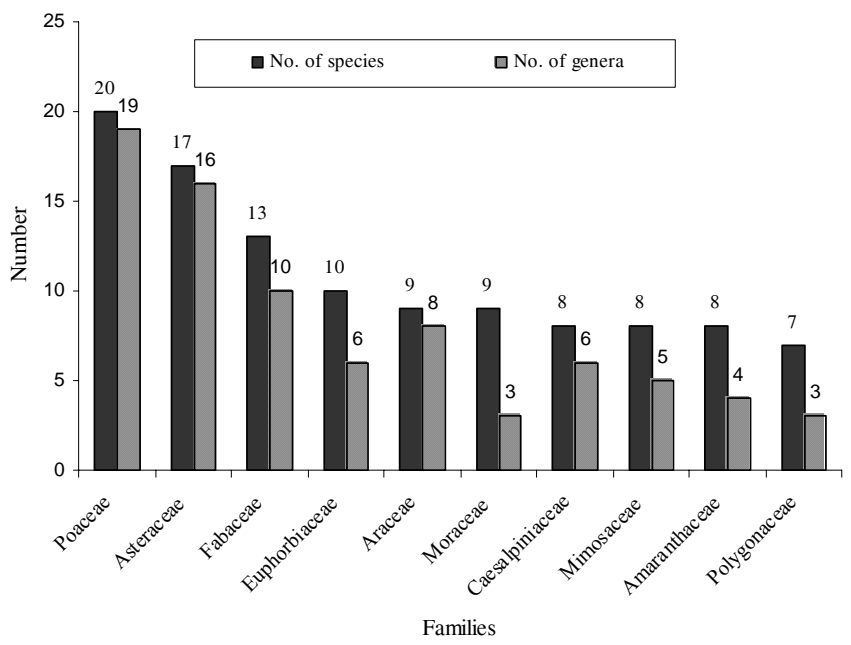

Fig. 1. Ten dominant plant families of Dhamrai Upazila.

Habit analysis shows that herbs are represented by 166 species including climbers, shrubs by 23 species and trees by 74 species. Out of 79 families recorded from the study area, 10 dominant families are Poaceae, Asteraceae, Fabaceae, Euphorbiaceae, Araceae, Moraceae, Caesalpiniaceae, Mimosaceae, Amaranthaceae and Polygonaceae. The dominant families along with the number of species and genera are shown in Fig. 1.

Ten dominant families comprising 109 species represent $41.44 \%$ and the remaining 69 families with a total of 154 species contribute $58.56 \%$. 
Table 1. Angiosperm species of Dhamrai Upazila.

\begin{tabular}{|c|c|c|c|c|}
\hline Taxa & $\begin{array}{l}\text { Bangla } \\
\text { name }\end{array}$ & Habit & Phenology & $\begin{array}{l}\text { Voucher } \\
\text { specimens }\end{array}$ \\
\hline \multicolumn{5}{|l|}{ MAGNOLIOPSIDA } \\
\hline \multicolumn{5}{|l|}{ 1. Annonaceae } \\
\hline 1. Annona reticulata $\mathrm{L}$. & Nona & Tree & F1 \& Fr: $10-1$ & R 50 \\
\hline 2. A. squamosa L. & Ata & Tree & F1 \& Fr: 3-12 & R 247 \\
\hline 3. Polyalthia longifolia (Sonn.) Thw. & Debdaru & Tree & F1 \& Fr: 3-10 & R 453 \\
\hline \multicolumn{5}{|l|}{ 2. Lauraceae } \\
\hline 4. Litsea glutinosa (Lour.) Roxb. & Menda & Tree & F1 \& Fr: 4-12 & R 109 \\
\hline \multicolumn{5}{|l|}{ 3. Piperaceae } \\
\hline 5. Peperomia pellucida (L.) H. B. \& K. & Luchipata & Herb & F1 \& Fr: $1-12$ & R 380 \\
\hline 6. Piper longum $\mathrm{L}$. & Pipul & Herb & Fl \& Fr: 6-9 & R 54 \\
\hline \multicolumn{5}{|l|}{ 4. Nymphaeaceae } \\
\hline 7. Nymphaea nouchali Burm. f. & Shapla & Herb & F1 \& Fr: $1-12$ & R 427 \\
\hline 8. N. pubescens Willd. & Sadashapla & Herb & F1 \& Fr: $1-12$ & R 334 \\
\hline 9. N. rubra Roxb. ex Andr. & Lalshapla & Herb & F1 \& Fr: $8-1$ & $\mathrm{R} 460$ \\
\hline \multicolumn{5}{|l|}{ 5. Ceratophyllaceae } \\
\hline 10. Ceratophyllum demersum L. & Jhanjhi & Herb & Fl \& Fr: 8-9 & R 420 \\
\hline \multicolumn{5}{|l|}{ 6. Menispermaceae } \\
\hline 11. Stephania japonica (Thunb.) Miers & Nimukha & Herb & Fl \& Fr: 3-9 & R 463 \\
\hline 12. Tinospora cordifolia Miers & Gulancho & Herb & F1 \& Fr: $1-10$ & R 373 \\
\hline \multicolumn{5}{|l|}{ 7. Ulmaceae } \\
\hline 13. Trema orientalis (L.) Blume & Jiban & Tree & F1 \& Fr: $9-10$ & R 325 \\
\hline \multicolumn{5}{|l|}{ 8. Moraceae } \\
\hline 14. Artocarpus lacucha Buch. & Deиa & Tree & F1 \& Fr: $2-9$ & R 335 \\
\hline 15. A. heterophyllus Lamk. & Kanthal & Tree & Fl \& Fr: 2-6 & R 391 \\
\hline 16. Ficus benghalensis L. & Bot & Tree & Fl \& Fr: 3-8 & R 390 \\
\hline 17. F. elastica Roxb. & Atabot & Tree & Fl \& Fr: $3-4$ & R 406 \\
\hline 18. F. hispida L. f. & Kakdumur & Tree & F1 \& Fr: 4-9 & R 43 \\
\hline 19. F. recemosa L. & Jagdumur & Tree & F1 \& Fr: 3-11 & R 59 \\
\hline 20. F. religiosa L. & Assawatha & Tree & F1 \& Fr: 5-9 & R 371 \\
\hline 21. F. rumphii Bl. & Hijulia & Tree & F1 \& Fr: 3-11 & R 258 \\
\hline 22. Streblus asper Lour. & Sheora & Tree & F1 \& Fr: 4-11 & R 34 \\
\hline \multicolumn{5}{|l|}{ 9. Urticaceae } \\
\hline 23. Laportea interrupta (L.) Chew & Bichuti & Shrub & Fl \& Fr: 5-9 & R 49 \\
\hline 24. Pilea microphylla (L.) Liebm & Latamaricha & Herb & F1 \& Fr: $1-12$ & R 461 \\
\hline 25. Pouzolazia zeylanica (L.) Benn. & Kullaruki & Herb & F1 \& Fr: 6-12 & R 158 \\
\hline \multicolumn{5}{|l|}{ 10. Casuarinaceae } \\
\hline 26. Casuarina equisetifolia Forst. & Jhau & Tree & Fl \& Fr: $11-2$ & R 218 \\
\hline \multicolumn{5}{|l|}{ 11. Nyctaginaceae } \\
\hline 27. Boerhavia repens L. & Punornava & Herb & Fl \& Fr: $1-12$ & R 24 \\
\hline \multicolumn{5}{|l|}{ 12. Chenopodiaceae } \\
\hline 28. Chenopodium album L. & Betua shak & Herb & F1 \& Fr: $12-3$ & R 187 \\
\hline 29. C. ambrosioides L. & Chandanbetu & Herb & F1 \& Fr: $11-3$ & R 195 \\
\hline
\end{tabular}


(Contd.)

\begin{tabular}{|c|c|c|c|c|}
\hline \multicolumn{5}{|l|}{ 13. Amaranthaceae } \\
\hline 30. Achyranthes aspera L. & Apang & Herb & Fl \& Fr: 9-4 & $\mathrm{R} 01$ \\
\hline 31. Alternanthera paronychioides St. Hil & Jhulikhata & Herb & Fl \& Fr: $1-5$ & $\mathrm{R} 93$ \\
\hline 32. A. philoxeroides (Mart.) Griseb. & Malancha shak & Herb & $\mathrm{Fl} \& \mathrm{Fr}: 3-6$ & R 419 \\
\hline 33. A. sessilis (L.) R. Br. & Chanchi & Herb & Fl \& Fr: $11-7$ & R 369 \\
\hline 34. Amaranthus spinosus L. & Kantanotey & Herb & Fl \& Fr: $1-12$ & R 08 \\
\hline 35. A. viridis $\mathrm{L}$. & Notey shak & Herb & F1 \& Fr: $12-4$ & R 130 \\
\hline 36. Centrostachys aquatica (R. Br.) Wall. & Not known & Herb & F1 \& Fr: $1-12$ & R 418 \\
\hline 37. Gomphrena celosioides L. & Botamphul & Herb & Fl \& Fr: 6-10 & R 102 \\
\hline \multicolumn{5}{|l|}{ 14. Portulacaceae } \\
\hline 38. Portulaca oleracea L. & Bara nunia & Herb & Fl \& Fr: 1-12 & R 398 \\
\hline \multicolumn{5}{|l|}{ 15. Polygonaceae } \\
\hline 39. Persicaria barbata (L.) Hara & Bekhunjabaj & Herb & F1 \& Fr: $8-4$ & R 434 \\
\hline 40. P. glabra (Willd.) Gomez & Bihagni & Herb & F1 \& Fr: 6-2 & $\mathrm{R} 262$ \\
\hline 41. P. hydropiper (L.) Spach. & Bishkatali & Herb & F1 \& Fr : 8-4 & R 39 \\
\hline 42. P. orientalis (L.) Spach. & Bara panimorich & Herb & F1 \& Fr: 3-8 & $\mathrm{R} 242$ \\
\hline 43. P. strigosa (R. Br.) Nakai & Kanta-bishkatali & Herb & F1 \& Fr: 5-1 & R 270 \\
\hline 44. Polygonum plebeium R. Br. & Anjaban & Herb & F1 \& Fr: $1-4$ & R 170 \\
\hline 45. Rumex maritimus L. & Banpalang & Herb & F1 \& Fr: $1-5$ & R 184 \\
\hline \multicolumn{5}{|l|}{ 16. Dilleniaceae } \\
\hline 46. Dillenia indica L. & Chalta & Tree & Fl \& Fr: $5-2$ & R 143 \\
\hline \multicolumn{5}{|l|}{ 17. Dipterocarpaceae } \\
\hline 47. Hopea odorata Roxb. & Telshur & Tree & F1 \& Fr: 2-6 & R 100 \\
\hline \multicolumn{5}{|l|}{ 18. Elaeocarpaceae } \\
\hline 48. Elaeocarpus floribundus B1. & Belphoi & Tree & F1 \& Fr: 5-6 & R 454 \\
\hline \multicolumn{5}{|l|}{ 19. Tiliaceae } \\
\hline 49. Corchorus aestuans L. & Janglipat & Herb & F1 \& Fr: 8-2 & R 279 \\
\hline 50. Triumfetta rhomboidea Jacq. & Banokra & Shrub & F1 \& Fr: $8-1$ & R 188 \\
\hline \multicolumn{5}{|l|}{ 20. Sterculiaceae } \\
\hline 51. Melochia corchorifolia L. & Tikiokra & Herb & F1 \& Fr: 3-6 & R 194 \\
\hline \multicolumn{5}{|l|}{ 21. Bombacaceae } \\
\hline 52. Bombax ceiba L. & Shimul & Tree & F1 \& Fr: $2-5$ & R 172 \\
\hline \multicolumn{5}{|l|}{ 22. Malvaceae } \\
\hline 53. Abutilon indicum (L.) Sweet & Potari & Herb & F1 \& Fr: 7-4 & R 332 \\
\hline 54. Sida acuta Burm. f. & Urusia & Herb & Fl \& Fr : 9-5 & R 299 \\
\hline 55. S. cordata (Burm. f.) Borss. & Pitberala & Herb & F1 \& Fr : 8-12 & R 12 \\
\hline 56. S. rhombifolia L. & Lalberela & Herb & Fl \& Fr: 7-12 & R 11 \\
\hline 57. Urena lobata L. & Jangli ghagra & Herb & F1 \& Fr: $10-4$ & R 28 \\
\hline \multicolumn{5}{|l|}{ 23. Lecythidaceae } \\
\hline 58. Barringtonia acutangula (L.) Gaertn. & Hizol & Tree & Fl \& Fr: $5-9$ & R 110 \\
\hline \multicolumn{5}{|l|}{ 24. Cucurbitaceae } \\
\hline 59. Coccinia grandis (L.) Voigt & Telakucha & Herb & F1 \& Fr: 3-11 & R 13 \\
\hline \multicolumn{5}{|l|}{ 25. Capparaceae } \\
\hline 60. Cleome rutidosperma DC. & Begunehurhurey & Herb & F1 \& Fr: $1-12$ & R 364 \\
\hline 61. C. viscosa $\mathrm{L}$. & Hurhuria & Herb & F1 \& Fr: $1-12$ & R 367 \\
\hline 62. Crateva magna (Lour.) DC. & Borun & Tree & F1 \& Fr: 2-4 & R 293 \\
\hline
\end{tabular}


(Contd.)

\begin{tabular}{|c|c|c|c|c|}
\hline \multicolumn{5}{|l|}{ 26. Brassicaceae } \\
\hline 63. Rorippa indica (L.) Hiern. & Bansarisha & Herb & F1 \& Fr: $1-6$ & R 234 \\
\hline \multicolumn{5}{|l|}{ 27. Moringaceae } \\
\hline 64. Moringa oleifera Lamk. & Sajna & Tree & Fl \& Fr: $10-3$ & R 117 \\
\hline \multicolumn{5}{|l|}{ 28. Ebenaceae } \\
\hline 65. Diospyros malabarica (Desr.) Kostel. & $G a b$ & Tree & Fl \& Fr: 5-8 & R 101 \\
\hline \multicolumn{5}{|l|}{ 29. Mimosaceae } \\
\hline 66. Acacia auriculiformis A. Cunn. & Akashmoni & Tree & Fl \& Fr: 6-2 & R 09 \\
\hline 67. A. mangium Willd. & Mangium & Tree & F1 \& Fr: 5-12 & R 472 \\
\hline 68. A. nilotica (L.) Delile & Babla & Tree & Fl \& Fr.: 8-5 & R 386 \\
\hline 69. Albizia lebbek (L.) Benth. & Kalokoroi & Tree & Fl \& Fr: 5-12 & R 292 \\
\hline 70. A. procera (Roxb.) Benth. & Koroi & Tree & F1 \& Fr: 5-12 & R 290 \\
\hline 71. Leucaena leucocephala (Lamk.) de Wit. & Ipil-ipil & Tree & F1 \& Fr: 3-11 & R 374 \\
\hline 72. Mimosa pudica $\mathrm{L}$. & Lajjabati & Herb & Fl \& Fr: $1-12$ & R 291 \\
\hline 73. Samanea saman (Jacq.) Merr. & Raintree & Tree & Fl. \& Fr: 4-10 & R 339 \\
\hline \multicolumn{5}{|l|}{ 30. Caesalpiniaceae } \\
\hline 74. Bauhinia acuminata L. & Kanchan & Shrub & Fl \& Fr: 5-7 & R 215 \\
\hline 75. Cassia fistula $\mathrm{L}$. & Sonalu & Tree & F1 \& Fr: 5-10 & R 219 \\
\hline 76. Delonix regia Rafin. & Krishnachura & Tree & Fl \& Fr: 4-9 & R 225 \\
\hline 77. Saraca asoca (Roxb.) Willd. & Ashok & Tree & Fl \& Fr: 2-6 & $\mathrm{R} 22$ \\
\hline 78. Senna sophera (L.) Roxb. & Chotokolkeshunda & Shrub & Fl \& Fr: $11-2$ & R 03 \\
\hline 79. S. occidentalis Roxb. & Bara kolkesunda & Shrub & Fl \& Fr: 5-10 & R 15 \\
\hline 80. S. tora (L.) Roxb. & Chakunda & Herb & Fl \& Fr: $1-12$ & R 14 \\
\hline 81. Tamarindus indica $\mathrm{L}$. & Tentul & Tree & F1 \& Fr: 4-12 & R 214 \\
\hline \multicolumn{5}{|l|}{ 31. Fabaceae } \\
\hline 82. Aeschynomene indica L. & Bhatshola & Herb & Fl \& Fr: 4-7 & R 244 \\
\hline 83. Alysicarpus heterophyllus (Baker.) Jafr. & Pannata & Herb & F1 \& Fr: 7-10 & R 454 \\
\hline 84. Butea monosperma (Lamk.) Taub. & Polash & Tree & Fl \& Fr: $1-4$ & R 88 \\
\hline 85. Canavalia ensiformis (L.) DC. & Makanshim & Herb & Fl \& Fr:11-3 & R 78 \\
\hline 86. Crotalaria pallida Ait. & Jhunjhuna & Herb & Fl \& Fr : 5-12 & $\mathrm{R} 42$ \\
\hline 87. Dalbergia sissoo Roxb. & Sisso & Tree & Fl \& Fr: 3-8 & R 41 \\
\hline 88. Desmodium gangeticum (L.) DC. & Salpani & Shrub & Fl \& Fr: 6-9 & R 259 \\
\hline 89. D. heterophyllum (Willd.) DC. & Not known & Herb & F1 \& Fr: 6-3 & R 20 \\
\hline 90. D. triflorum (L.) DC. & Kodaliya & Herb & Fl \& Fr: $1-12$ & R 27 \\
\hline 91. Erythrina veriegata $\mathrm{L}$. & Mandar & Tree & Fl \& Fr: $2-5$ & R 456 \\
\hline 92. Melilotus alba Lamk. & Sadabanmethi & Herb & F1 \& Fr: 3-10 & R 256 \\
\hline 93. Sesbania cannabina (Retz.) Poir. & Dhanchi & Herb & Fl \& Fr: 7-12 & R 457 \\
\hline 94. S. grandiflora (L.) Poir. & Bakphul & Shrub & Fl \& Fr: $12-3$ & R 256 \\
\hline \multicolumn{5}{|l|}{ 32. Lythraceae } \\
\hline 95. Ammannia multiflora Roxb. & Not known & Herb & Fl \& Fr : 7-9 & R 330 \\
\hline \multicolumn{5}{|l|}{ 33. Myrtaceae } \\
\hline 96. Eucalyptus citriodora Hook. & Eucalyptus & Tree & F1 \& Fr: $1-12$ & R 308 \\
\hline 97. Psidium guajava $\mathrm{L}$. & Peyara & Tree & F1 \& Fr: $1-12$ & R 328 \\
\hline 98. Syzygium cumini (L.) Skeels & Jam & Tree & Fl \& Fr: 3-6 & R 376 \\
\hline
\end{tabular}




\begin{tabular}{|c|c|c|c|c|}
\hline \multicolumn{5}{|l|}{ 34. Punicaceae } \\
\hline 99. Punica granatum L. & Dalim & Tree & Fl \& Fr: $1-12$ & R 165 \\
\hline \multicolumn{5}{|l|}{ 35. Onagraceae } \\
\hline 100. Ludwigia adscendens (L.) Hara & Kesardam & Herb & Fl \& Fr: 3-12 & R 21 \\
\hline 101. L. hyssopifolia (G. Don) Exell & Not known & Herb & F1 \& Fr: $1-12$ & R 407 \\
\hline 102. L. prostrata Roxb. & Not known & Herb & Fl \& Fr: $1-9$ & R 152 \\
\hline \multicolumn{5}{|l|}{ 36. Combretaceae } \\
\hline $\begin{array}{l}\text { 103. Terminalia arjuna (Roxb. ex DC.) } \\
\text { Wight \& Arn. }\end{array}$ & Arjun & Tree & Fl \& Fr: 4-10 & R 171 \\
\hline 104. T. bellirica (Gaertn.) Roxb. & Bohera & Tree & F1 \& Fr: 3-11 & R 134 \\
\hline 105. T. chebula Retz. & Haritaki & Tree & F1 \& Fr: 4-10 & R 186 \\
\hline \multicolumn{5}{|l|}{ 37. Euphorbiaceae } \\
\hline 106. Acalypha indica L. & Muktajhuri & Herb & F1 \& Fr: $12-4$ & R 455 \\
\hline 107. Croton bonplandianus Baill. & Croton & Herb & Fl \& Fr: 4-9 & R 04 \\
\hline 108. Euphorbia hirta L. & Dudhiya & Herb & Fl \& Fr: 2-3 & R 06 \\
\hline 109. E. thymifolia L. & Swetkerui & Herb & F1 \& Fr: $1-12$ & R 79 \\
\hline 110. Phyllanthus acidus (L.) Skeels & Orbori & Tree & F1 \& Fr: 3-6 & $\mathrm{R} 80$ \\
\hline 111. P. emblica L. & Amlaki & Tree & F1 \& Fr: 3-9 & R 94 \\
\hline 112. P. niruri L. & Bhuiamla & Herb & F1 \& Fr: 8-10 & R 02 \\
\hline 113. P. reticulatus Poir. & Chitki & Shrub & F1 \& Fr: 6-10 & $\mathrm{R} 18$ \\
\hline 114. Ricinus communis L. & Rerhi & Shrub & F1 \& Fr: 9-2 & R 149 \\
\hline 115. Trewia nudiflora $\mathrm{L}$. & Meragoda & Tree & F1 \& Fr: 5-10 & R 348 \\
\hline \multicolumn{5}{|l|}{ 38. Rhamnaceae } \\
\hline 116. Ziziphus mauritiana Lamk. & Boroi & Tree & F1 \& Fr : 6-2 & R 378 \\
\hline \multicolumn{5}{|l|}{ 39. Vitaceae } \\
\hline 117. Tetrastigma angustifolia (Roxb.) Planch. & Not known & Herb & F1 \& Fr: 3-6 & R 62 \\
\hline \multicolumn{5}{|l|}{ 40. Sapindaceae } \\
\hline 118. Cardiospermum halicacabum L. & Phutka & Herb & Fl \& Fr: 4-6 & R 58 \\
\hline 119. Litchi chinensis Sonn. & Lichu & Tree & F1 \& Fr: 4-6 & R 115 \\
\hline \multicolumn{5}{|l|}{ 41. Anacardiaceae } \\
\hline 120. Lannea coromandelica (Houtt.) Merr. & Jiga & Tree & Fl \& Fr: $12-5$ & R 429 \\
\hline 121. Mangifera indica L. & $A m$ & Tree & Fl \&Fr: 1-6 & R 452 \\
\hline \multicolumn{5}{|l|}{ 42. Meliaceae } \\
\hline $\begin{array}{l}\text { 122. Aphanamixis polystachya (Wall.) R.N. } \\
\text { Parker }\end{array}$ & Royna & Tree & F1 \& Fr: $2-5$ & R 202 \\
\hline 123. Azadirachta indica A. Juss. & Nim & Tree & F1 \& Fr: 3-7 & R 392 \\
\hline 124. Melia azedarach L. & Goranim & Tree & F1 \& Fr: 3-2 & R 17 \\
\hline 125. Swietenia mahagoni Jacq. & Mehogoni & Tree & F1 \& Fr: 4-10 & R 122 \\
\hline \multicolumn{5}{|l|}{ 43. Rutaceae } \\
\hline 126. Aegle marmelos (L.) Correa & Bel & Tree & Fl \& Fr: 5-7 & R 118 \\
\hline 127. Citrus limon (L.) Burm. & Goralebu & Tree & F1 \& Fr: 3-11 & R 167 \\
\hline 128. Glycosmis pentaphylla (Retz.) A. DC. & Datmajan & Tree & F1 \& Fr: 2-4 & R 263 \\
\hline 129. Limonia acidissima $\mathrm{L}$. & Koethbel & Tree & F1 \& Fr: 2-12 & R 229 \\
\hline \multicolumn{5}{|l|}{ 44. Oxalidaceae } \\
\hline 130. Averrhoa carambola L & Kamranga & Tree & F1 \& Fr: $10-7$ & R 361 \\
\hline 131. Oxalis corniculata L. & Amrul & Herb & F1 \& Fr: 9-5 & R 95 \\
\hline
\end{tabular}


(Contd.)

\begin{tabular}{|c|c|c|c|c|}
\hline \multicolumn{5}{|l|}{ 45. Apiaceae } \\
\hline 132. Centella asiatica (L.) Urban & Thankuni & Herb & F1 \& Fr: 4-10 & R 135 \\
\hline 133. Oenanthe benghalensis Benth. & Panturasi & Herb & F1 \& Fr: 4-8 & R 458 \\
\hline \multicolumn{5}{|l|}{ 46. Apocynaceae } \\
\hline 134. Alstonia scholaris (L.) R. Br. & Chatim & Tree & F1 \& Fr: $11-5$ & R 193 \\
\hline 135. Nerium oleander L. & Raktakarobi & Tree & Fl \& Fr: $1-7$ & R 189 \\
\hline 136. Plumeria obtusa L. & Gorurchampa & Tree & F1 \& Fr: 5-9 & R 183 \\
\hline 137. Tabernaemontana divaricata (L.) R. Br. & Dudhphul & Shrub & Fl \& Fr: 5-12 & R 113 \\
\hline 138. Thevetia peruviana (Pers.) K. Schum. & Holdekarobi & Tree & F1 \& Fr: $1-12$ & R 114 \\
\hline \multicolumn{5}{|l|}{ 47. Asclepiadaceae } \\
\hline 139. Calotropis gigantea (L.) R. Br. & Bara akondo & Shrub & F1 \& Fr: $1-12$ & R 331 \\
\hline \multicolumn{5}{|l|}{ 48. Solanaceae } \\
\hline 140. Datura metel L. & Dhutra & Shrub & F1 \& Fr: 4-9 & R 129 \\
\hline 141. Physalis angulata $\mathrm{L}$. & Bantepari & Herb & F1 \& Fr: 2-8 & R 156 \\
\hline 142. Solanum indicum $\mathrm{L}$. & Titbegun & Herb & F1 \& Fr: $1-12$ & R 274 \\
\hline 143. S. nigrum L. & Kakmachi & Herb & Fl \& Fr : $1-12$ & R 150 \\
\hline 144. S. torvum Sw. & Gothbegun & Shrub & F1 \& Fr: $1-12$ & R 169 \\
\hline \multicolumn{5}{|l|}{ 49. Convolvulaceae } \\
\hline 145. Evolvulus nummularius (L.) L. & Bhuiokra & Herb & F1 \& Fr: $1-12$ & R 357 \\
\hline 146. Ipomoea aquatica Forssk. & Kalmishak & Herb & F1 \& Fr: $10-2$ & R 83 \\
\hline 147. I. fistulosa Mart. ex Choisy & Dholkalmi & Shrub & F1 \& Fr: $1-12$ & R 415 \\
\hline \multicolumn{5}{|l|}{ 50. Cuscutaceae } \\
\hline 148. Cuscuta reflexa Roxb. & Sharnalata & Herb & F1 \& Fr: $1-4$ & R 166 \\
\hline \multicolumn{5}{|l|}{ 51. Menyanthaceae } \\
\hline $\begin{array}{l}\text { 149. Nymphoides hydrophylla (Lour.) O. } \\
\text { Kuntze }\end{array}$ & Chandmala & Herb & F1 \& Fr: $10-2$ & R 425 \\
\hline 150. N. indicum (L.) O. Kuntze & Panchuli & Herb & F1 \& Fr: $10-2$ & R 424 \\
\hline \multicolumn{5}{|l|}{ 52. Hydrophyllaceae } \\
\hline 151. Hydrolea zeylanica (L.) Vahl & Islanguli & Herb & F1 \& Fr: $10-3$ & R 458 \\
\hline \multicolumn{5}{|l|}{ 53. Boraginaceae } \\
\hline 152. Heliotropium indicum L. & Hatishur & Herb & F1 \& Fr: $1-12$ & R 266 \\
\hline 153. H. ovalifolium Forssk. & Nagaphuli & Herb & F1 \& Fr: 2-8 & R 267 \\
\hline \multicolumn{5}{|l|}{ 54. Verbenaceae } \\
\hline 154. Clerodendrum viscosum Vent & Bhat & Herb & F1 \& Fr: $12-6$ & R 174 \\
\hline 155. Gmelina arborea Roxb. & Gamari & Tree & F1 \& Fr: 2-9 & R 416 \\
\hline 156. Lantana camara L. & Lantana & Shrub & F1 \& Fr: $1-12$ & R 382 \\
\hline 157. Lippia alba (Mill.) Briton & Pichas-lakri & Shrub & F1 \& Fr: $1-12$ & R 245 \\
\hline 158. Phyla nodiflora (L.) Greene & Bhuiokra & Herb & F1 \& Fr: $1-12$ & R 141 \\
\hline 159. Tectona grandis L. & Shegun & Tree & F1 \& Fr: 7-11 & R 103 \\
\hline 160. Vitex negundo L. & Nishinda & Tree & F1 \& Fr: 4-2 & R 62 \\
\hline \multicolumn{5}{|l|}{ 55. Lamiaceae } \\
\hline 161. Hyptis suaveolens (L.) Poit. & Thukma & Herb & Fl. \& Fr: 11-4 & R 462 \\
\hline 162. Leonurus sibiricus L. & Roktodhron & Herb & F1 \& Fr: $1-12$ & R 30 \\
\hline 163. Leucus aspera (Willd.) Link & Dhondokalas & Herb & F1 \& Fr: 1-12 & R 29 \\
\hline 164. Ocimum americanum L. & Bantulshi & Herb & F1 \& Fr: 6-2 & R 107 \\
\hline 165. O. tenuiflorum L. & Tulshi & Herb & F1 \& Fr: 11-2 & R 128 \\
\hline
\end{tabular}


(Contd.)

\begin{tabular}{|c|c|c|c|c|}
\hline \multicolumn{5}{|l|}{ 56. Scrophulariaceae } \\
\hline 166. Limnophila heterophylla Roxb. & Not known & Herb & F1 \& Fr: $2-9$ & R 437 \\
\hline 167. Lindernia anagallis (Burm. f.) Pennel. & Not known & Herb & F1 \& Fr: 3-11 & R 315 \\
\hline 168. L. crustacea (L.) F. Muell. & Not known & Herb & F1 \& Fr: 4-12 & R 220 \\
\hline 169. L. rotundifolia (L.) Alston & Not known & Herb & F1 \& Fr: $1-12$ & R 393 \\
\hline 170. Mecardonia procumbens (Mill.) Small & Not known & Herb & F1 \& Fr: 2-6 & R 319 \\
\hline 171. Scoparia dulcis L. & Bondhoney & Herb & F1 \& Fr: $12-1$ & R 81 \\
\hline \multicolumn{5}{|l|}{ 57. Acanthaceae } \\
\hline 172. Dipteracanthus prostratus (Poir.) Nees & Not known & Herb & F1 \& Fr: $5-8$ & R 366 \\
\hline $\begin{array}{l}\text { 173. Hygrophila schulli (Buch.-Ham.) M.R. } \\
\text { \& S.N. Almeida }\end{array}$ & Talmakhna & Herb & F1 \& Fr: $10-1$ & R 417 \\
\hline 174. Justicia adhatoda L. & Basak & Shrub & F1 \& Fr: $1-4$ & R 132 \\
\hline 175. J. gendarussa L. & Jagadmadan & Shrub & F1 \& Fr: $12-5$ & R 145 \\
\hline 176. Ruellia tuberosa L. & Chatpotey & Herb & F1 \& Fr: $1-2$ & R 253 \\
\hline 177. Rungia pectinata (L.) Nees & Pindi & Herb & F1 \& Fr: 11-5 & R 142 \\
\hline \multicolumn{5}{|l|}{ 58. Bignoniaceae } \\
\hline 178. Tecoma stans (L.) Juss. ex Kunth & Holde Tecoma & Shrub & Fl \& Fr: 5-12 & R 85 \\
\hline \multicolumn{5}{|l|}{ 59. Lentibulariaceae } \\
\hline 179. Utricularia aurea Lour. & Jhangi & Herb & F1 \& Fr: $7-4$ & R 423 \\
\hline \multicolumn{5}{|l|}{ 60. Campanulaceae } \\
\hline $\begin{array}{l}\text { 180. Wahlenbergia erecta (Roth ex Roem. \& } \\
\text { Schult.) Tuya }\end{array}$ & Not known & Herb & F1 \& Fr: $10-2$ & R 223 \\
\hline \multicolumn{5}{|l|}{ 61. Rubiaceae } \\
\hline 181. Dentella repens Forst. & Bhuipat & Herb & Fl. \& Fr: $8-9$ & R 33 \\
\hline 182. Hedyotis corymbosa (L.) Lamk. & Khetpapra & Herb & Fl \& Fr: $1-12$ & R 127 \\
\hline 183. Neolamarckia cadamba (Roxb.) Bosser. & Kadam & Tree & F1 \& Fr: 5-8 & R 397 \\
\hline \multicolumn{5}{|l|}{ 62. Asteraceae } \\
\hline 184. Ageratum conyzoides L. & Fulkuri & Herb & F1 \& Fr: 9-6 & R 154 \\
\hline 185. Blumea lacera (Burm. f.) DC. & Kukurshunga & Herb & F1 \& Fr: $11-7$ & $\mathrm{R} 48$ \\
\hline 186. B. membranacea Wall. ex DC. & Not known & Herb & F1 \& Fr: $1-3$ & R 69 \\
\hline 187. Chromolaena odorata (L.) King \& Robin. & German lata & Shrub & F1 \& Fr: $12-4$ & R 180 \\
\hline 188. Cirsium arvense (L.) Scop. & Shial-kata & Herb & F1 \& Fr: 2-6 & R 31 \\
\hline $\begin{array}{l}\text { 189. Cotula hemispherica (Roxb.) Wall. ex } \\
\text { Clarke }\end{array}$ & Babuni & Herb & F1 \& Fr: $12-3$ & R 207 \\
\hline 190. Eclipta alba (L.) Hassk. & Kalokeshi & Herb & F1 \& Fr: 1-12 & R 148 \\
\hline 191. Enhydra fluctuans Lour. & Helencha & Herb & F1 \& Fr: $1-4$ & R 421 \\
\hline 192. Gnaphalium luteo-album L. & Bara kamra & Herb & F1 \& Fr: $3-8$ & R 180 \\
\hline 193. Grangea maderaspatana (L.) Poir. & Nemuti & Herb & F1 \& Fr: $12-5$ & R 233 \\
\hline 194. Mikania cordata (Burm. f.) Robinson & Assam lata & Herb & Fl \& Fr: $10-2$ & R 51 \\
\hline 195. Spilanthes calva DC. & Marhatitiga & Herb & Fl \& Fr: 1-12 & R 53 \\
\hline 196. Synedrella nodiflora (L.) Gaertn. & Not known & Herb & Fl \& Fr: $1-12$ & R 19 \\
\hline 197. Tridax procumbens L. & Tridhara & Herb & F1 \& Fr: 1-12 & R 301 \\
\hline 198. Vernonia cinerea (L.) Nees & Kukshim & Herb & F1 \& Fr: 5-9 & R 124 \\
\hline 199. Xanthium indicum Koen. & Ghagra & Herb & F1 \& Fr: 1-12 & R 35 \\
\hline 200. Youngia japonica (L.) DC. & Not known & Herb & F1 \& Fr: 8-1 & R 155 \\
\hline
\end{tabular}


(Contd.)

\begin{tabular}{|c|c|c|c|c|}
\hline \multicolumn{5}{|l|}{ LILIOPSIDA } \\
\hline \multicolumn{5}{|l|}{ 63. Limnocharitaceae } \\
\hline 201. Limnocharis flava (L.) Buchen. & Not known & Herb & F1 \& Fr: 6-9 & R 414 \\
\hline \multicolumn{5}{|l|}{ 64. Alismataceae } \\
\hline $\begin{array}{l}\text { 202. Sagittaria guayanensis H. B. \& K. subsp. } \\
\text { lappula (D. Don) Bogin }\end{array}$ & Kaowathukri & Herb & F1 \& Fr: 6-9 & $\mathrm{R} 468$ \\
\hline 203. S. sagittifolia L. & Chhotokut & Herb & Fl \& Fr: $10-12$ & R 349 \\
\hline \multicolumn{5}{|l|}{ 65. Hydrocharitaceae } \\
\hline 204. Hydrilla verticillata (L. f.) Royle & Kureli & Herb & Fl \& Fr: 1-12 & R 443 \\
\hline 205. Ottelia alismoides (L.) Pers. & Panikola & Herb & F1 \& Fr: 1-12 & $\mathrm{R} 446$ \\
\hline 206. Vallisneria spiralis L. & Patajhangi & Herb & Fl \& Fr: $10-3$ & $\mathrm{R} 451$ \\
\hline \multicolumn{5}{|l|}{ 66. Aponogetonaceae } \\
\hline 207. Aponogeton natans (L.) Engl. \& Krause & Ghentu & Herb & F1 \& Fr: 4-10 & R 37 \\
\hline \multicolumn{5}{|l|}{ 67. Arecaceae } \\
\hline 208. Areca catechu L. & Supari & Tree & $\mathrm{F} 1 \& \mathrm{Fr}: 1-12$ & $\mathrm{R} 64$ \\
\hline 209. Borassus flabellifer L. & Tal & Tree & F1 \& Fr: $1-10$ & R 67 \\
\hline 210. Calamus viminalis Willd. & Bara bet & Shrub & Fl \& Fr: 1-12 & R 217 \\
\hline 211. Cocos nucifera $\mathrm{L}$. & Narikel & Tree & Fl \& Fr: $3-7$ & R 68 \\
\hline 212. Phoenix sylvestris Roxb. & Khejur & Tree & F1 \& Fr: $12-6$ & R 314 \\
\hline \multicolumn{5}{|l|}{ 68. Pandanaceae } \\
\hline 213. Pandanus odorus Ridl. & Polao pata & Shrub & Fl \& Fr: 5-10 & $\mathrm{R} 470$ \\
\hline \multicolumn{5}{|l|}{ 69. Araceae } \\
\hline 214. Alocasia cucullata (Lour.) G. Don & Bishkachu & Herb & F1 \& Fr: 5-7 & R 356 \\
\hline 215. A. macrorrhizos (L.) G. Don & Mankachu & Herb & F1 \& Fr: 7-10 & R 60 \\
\hline 216. Amorphophallus campanulatus Decne & Olkachu & Herb & F1 \& Fr: 5-11 & $\mathrm{R} 40$ \\
\hline 217. Colocasia esculenta (L.) Schott & Kochu & Herb & $\mathrm{F} 1 \& \mathrm{Fr}: 5-10$ & $\mathrm{R} 250$ \\
\hline 218. Lasia spinosa (L) Thw. & Kantakachu & Herb & F1 \& Fr: 1-11 & R 354 \\
\hline 219. Pistia stratiotes L. & Topapana & Herb & F1 \& Fr: $10-3$ & $\mathrm{R} 440$ \\
\hline 220. Rhaphidophora aurea (Lindl. \& Andr.) Birds. & Not known & Herb & F1 \& Fr: 4-5 & $\mathrm{R} 286$ \\
\hline 221. Syngonium podophyllum Schott & Not known & Herb & F1 \& Fr: $2-11$ & R 369 \\
\hline 222. Typhonium trilobatum (L.) Schott & Ghetkachu & Herb & Fl \& Fr: 4-10 & R 280 \\
\hline \multicolumn{5}{|l|}{ 70. Lemnaceae } \\
\hline 223. Lemna perpusilla Torrey & Khudipana & Herb & F1 \& Fr: 5-9 & R 469 \\
\hline \multicolumn{5}{|l|}{ 71. Commelinaceae } \\
\hline 224. Commelina benghalensis L. & Kanchira & Herb & Fl \& Fr: 2-12 & $\mathrm{R} 409$ \\
\hline 225. C. longifolia Lamk. & Panikanchira & Herb & F1 \& Fr: $12-1$ & R 98 \\
\hline 226. Cyanotis cristata (L.) D. Don & Not known & Herb & F1 \& Fr: 9-2 & R 297 \\
\hline 227. Murdania nodiflora (L.) Brenan & Kureli & Herb & F1 \& Fr: 7-9 & R 399 \\
\hline \multicolumn{5}{|l|}{ 72. Cyperaceae } \\
\hline 228. Cyperus exaltatus Retz. & Not known & Herb & Fl \& Fr: $10-3$ & R 182 \\
\hline 229. C. iria L. & Bara chancha & Herb & F1 \& Fr: 5-10 & $\mathrm{R} 252$ \\
\hline 230. C. rotundus L. & Mutha & Herb & F1 \& Fr: 5-9 & R 237 \\
\hline 231. Eleocharis ochrastachys Steud. & Not known & Herb & F1 \& Fr: 9-1 & R 17 \\
\hline $\begin{array}{l}\text { 232. Kyllinga nemoralis (J.R. Forst \& G. Forst.) } \\
\text { Dandy }\end{array}$ & Nirbishi & Herb & F1 \& Fr: 6-9 & R 273 \\
\hline
\end{tabular}


(Contd.)

\begin{tabular}{|c|c|c|c|c|}
\hline 233. Pycreus pumilus (L.) Nees ex Clarke & Not known & Herb & Fl.\& Fr: 4-9 & R 182 \\
\hline 234. Schoenoplectus articulatus (L.) Palla & Potpote & Herb & Fl \& Fr: $10-3$ & $\mathrm{R} 75$ \\
\hline \multicolumn{5}{|l|}{ 73. Poaceae } \\
\hline 235. Bambusa balcooa Roxb. & Barakbans & Tree & F1 \& Fr: 3-9 & R 346 \\
\hline 236. B. bambos (L.) Voss & Bans & Tree & Fl \& Fr: 4-3 & $\mathrm{R} 233$ \\
\hline 237. Chrysopogon aciculatus (Retz.) Trin. & Premkanta & Herb & Fl \& Fr: $1-12$ & $\mathrm{R} 272$ \\
\hline 238. Coix lacryma-jobi L. & Tasbi & Shrub & F1 \& Fr: $8-3$ & R 471 \\
\hline 239. Cynodon dactylon (L.) Pers. & Durba & Herb & Fl \& Fr: 7-12 & $\mathrm{R} 77$ \\
\hline 240. Dactyloctenium aegyptium (L.) P. Beauv. & Makra & Herb & Fl \& Fr: $1-12$ & $\mathrm{R} 227$ \\
\hline 241. Digitaria sanguinalis (L.) Scop. & Makunjali & Herb & Fl \& Fr: 8-10 & R 381 \\
\hline 242. Echinochloa crusgalli (L.) P. Beauv. & Barashymaghas & Herb & Fl \& Fr: 4-10 & R 249 \\
\hline 243. Eleusine indica (L.) Gaertn. & Malankuri & Herb & Fl \& Fr: $6-8$ & R 111 \\
\hline 244. Eragrostis tenella (L.) P. Beauv. & Koni & Herb & Fl \& Fr: 3-9 & R 182 \\
\hline 245. Hygroryza aristata (Retz.) Nees & Phutki & Herb & Fl \& Fr: $10-2$ & $\mathrm{R} 442$ \\
\hline 246. Imperata cylindrica (L.) P. Beauv. & Kash & Herb & Fl \& Fr: $10-1$ & R 112 \\
\hline 247. Oplismenus burmanii (Rtez.) P. Beauv. & Not known & Herb & Fl \& Fr: 9-1 & R 379 \\
\hline 248. Ottochloa nodosa (Kunth.) Dandy & Not known & Herb & Fl \& Fr: 7-12 & R 177 \\
\hline 249. Paspalidium punctatum (Burm.) A. Camus & Petinar & Herb & Fl \& Fr: $8-3$ & R 126 \\
\hline 250. Paspalum longifolium Roxb. & Not known & Herb & Fl \& Fr: 5-8 & R 248 \\
\hline 251. Saccharum spontaneam L. & Kash & Herb & Fl \& Fr: $1-12$ & $\mathrm{R} 282$ \\
\hline 252. Setaria glauca (L.) P. Beauv. & Kauni & Herb & Fl \& Fr: 9-12 & R 190 \\
\hline 253. Sporolobus diander (Retz.) P. Beauv. & Benajoni & Herb & Fl \& Fr: 3-10 & R 250 \\
\hline 254. Vetiveria zizanioides (L.) Nash & Khuskhus & Herb & Fl \& Fr: 9-12 & R 206 \\
\hline \multicolumn{5}{|l|}{ 74. Zingiberaceae } \\
\hline 255. Curcuma zedoaria (Christm.) Rosc. & Shoti & Herb & Fl \& Fr: 2-7 & $\mathrm{R} 235$ \\
\hline \multicolumn{5}{|l|}{ 75. Costaceae } \\
\hline 256. Costus speciosus Sm. & Kushtha & Herb & Fl \& Fr: 4-10 & R 398 \\
\hline \multicolumn{5}{|l|}{ 76. Pontederiaceae } \\
\hline 257. Eichhornia crassipes (Mart.) Solms & Kochuripana & Herb & Fl \& Fr: $1-12$ & $\mathrm{R} 448$ \\
\hline 258. Monochoria hastata (L.) Solms & Baranukha & Herb & Fl \& Fr: $1-12$ & $\mathrm{R} 447$ \\
\hline 259. M. vaginalis (Burm. f.) Presl. & Nukha & Herb & Fl \& Fr: 5-1 & $\mathrm{R} 449$ \\
\hline \multicolumn{5}{|l|}{ 77. Liliaceae } \\
\hline 260. Curculigo orchidioides Gaertn. & Tali & Herb & Fl \& Fr: 2-8 & $\mathrm{R} 413$ \\
\hline 261. Pancratium verecandum Ait. & Kumur & Herb & F1 \& Fr: 3-7 & R 99 \\
\hline \multicolumn{5}{|l|}{ 78. Dioscoreaceae } \\
\hline 262. Dioscorea alata L. & Guraniya alu & Herb & F1 \& Fr: $10-12$ & $\mathrm{R} 75$ \\
\hline \multicolumn{5}{|l|}{ 79. Orchidaceae } \\
\hline 263. Vanda tessellata (Roxb.) Hook. & Rasna & Herb & Fl \& Fr: 4-9 & R 240 \\
\hline
\end{tabular}

Aquatic vegetation: Dhamrai Upazila is endowed with different aquatic habitats including ponds, tanks, beels and other low-lying areas filled with seasonal water. This habitat is rich with diverse aquatic vegetation. Some of the important aquatic angiosperms are Alternanthera philoxeroides, Centrostachys aquatica, Ceratophyllum demersum, Eichhornia crassipes, Enhydra fluctuans, Hydrilla verticillata, Hygrophila auriculata, Hygroryza aristata, Ipomoea aquatica, Lemna purpusilla, Limnophila heterophylla, Ludwigia adscendens, Monochria hastata, M. vaginalis, Nymphaea nouchali, N. pubescens, Nymphoides indicum, Ottelia alismoides, Pistia stratiotes, Sagittaria guayanensis subsp. lappula, S. sagittifolia, Schoenoplectus articulatus, Utricularia aurea and Vallisneria spiralis. 
Medicinally important plants: The present study identifies 62 medicinal plants locally used by the people of Dhamrai area for the treatment of at least 30 common diseases and some of the important diseases are diabetes, jaundice, diarrhoea, dysentery, cold and cough, asthma, fever, spleen and liver complaints, piles, ulcers and several skin diseases. The medicinal plants are listed in Table 2 along with their family names, part(s) used and the diseases treated for. Photographs of some wild medicinally important species are presented in Plate 1.

Table 2. Medicinal plants used by the local people of Dhamrai area.

\begin{tabular}{|c|c|c|c|}
\hline $\begin{array}{l}\text { Plant } \\
\text { species }\end{array}$ & $\begin{array}{l}\text { Family } \\
\text { name }\end{array}$ & $\begin{array}{l}\text { Part(s) } \\
\text { used }\end{array}$ & $\begin{array}{l}\text { Diseases to } \\
\text { be treated }\end{array}$ \\
\hline 1. Achyranthes aspera L. & Amaranthaceae & $\mathrm{R}$ & Jaundice \\
\hline 2. Aegle marmelos (L.) Correa & Rutaceae & Fr, $\mathrm{R}$ & Diarrhoea and dysentery \\
\hline 3. Ageratum conyzoides L. & Asteraceae & St, L, R & Fever, chronic ulcers and pneumonia \\
\hline 4. Albizia procera (Roxb.) Benth. & Mimosaceae & $\mathrm{B}, \mathrm{L}$ & Insecticide and fish poisoning \\
\hline 5. Alstonia scholaris (L.) R. Br. & Apocynaceae & $\mathrm{B}$ & Fever and asthma \\
\hline 6. Amaranthus spinosus L. & Amaranthaceae & L, St, R & $\begin{array}{l}\text { Blood purifier, rheumatism and } \\
\text { irregular menstruation }\end{array}$ \\
\hline 7. Amaranthus viridis $\mathrm{L}$. & Amaranthaceae & WP & Snake-bite \\
\hline 8. Annona squamosa L. & Annonaceae & $\mathrm{B}$ & Diarrhoea \\
\hline $\begin{array}{l}\text { 9. Aphanamixis polystachya (Wall.) R. } \\
\text { N. Parker }\end{array}$ & Meliaceae & $\mathrm{B}$ & Spleen and lever diseases \\
\hline 10. Averrhoa carambola L. & Oxalidaceae & $\mathrm{Fr}$ & Piles \\
\hline 11. Azadirachta indica A. Juss. & Meliaceae & $\mathrm{L}, \mathrm{B}$ & $\begin{array}{l}\text { Fever, sores, spleen complaints and } \\
\text { small pox }\end{array}$ \\
\hline 12. Blumea lacera (Burm. f.) DC. & Asteraceae & $\mathrm{R}$ & Mouth diseases \\
\hline 13. Boerhavia repens L. & Nyctaginaceae & $\mathrm{R}, \mathrm{L}$ & Diuretic, laxative and jaundice \\
\hline 14. Butea monosperma (Lamk.) Taub. & Fabaceae & $\mathrm{Fl}$ & Diarrhoea \\
\hline 15. Centella asiatica (L.) Urban & Apiaceae & WP & Dysentery and ulcer \\
\hline 16. Clerodendrum viscosum Vent & Verbenaceae & $\mathrm{L}, \mathrm{R}$ & Tumors, skin diseases and snake-bite \\
\hline 17. Coccinia grandis (L.) Voigt & Cucurbitaceae & $\mathrm{L}$ & Diabetes and skin diseases \\
\hline 18. Colocasia esculenta (L.) Schott & Araceae & $\mathrm{C}, \mathrm{L}$ & $\begin{array}{l}\text { Scorpion bites, astringent and } \\
\text { stimulant }\end{array}$ \\
\hline 19. Commelina benghalensis L. & Commelinaceae & WP & Itches, sores and urinary burning \\
\hline 20. Cuscuta reflexa Roxb. & Cuscutaceae & $\mathrm{S}$ & Jaundice \\
\hline 21. Cynodon dactylon (L.) Pers. & Poaceae & WP & Stop bleeding and tooth-ache \\
\hline 22. Cyperus rotundus $\mathrm{L}$. & Cyperaceae & $\mathrm{R}$ & Ulcer, sores and urinary problems \\
\hline 23. Dillenia indica L. & Dilleniaceae & $\mathrm{Fr}$ & Diarrhoea and dysentery \\
\hline 24. Eclipta alba (L.) Hassk. & Asteraceae & WP & Skin diseases and jaundice \\
\hline 25. Eleusine indica (L.) Gaertn. & Poaceae & WP & Liver complaints \\
\hline 26. Enhydra fluctuans Lour. & Asteraceae & $\mathrm{L}$ & $\begin{array}{l}\text { Bronchitis, biliousness and } \\
\text { leucoderma }\end{array}$ \\
\hline 27. Ficus hispida L. f. & Moraceae & $\mathrm{Fr}$ & Diabetes \\
\hline 28. F. religiosa L. & Moraceae & $\mathrm{B}$ & Skin diseases and tooth-ache \\
\hline 29. Glycosmis pentaphylla (Retz.) DC. & Rutaceae & $\mathrm{L}, \mathrm{St}$ & Jaundice and tooth-ache \\
\hline 30. Heliotropium indicum $\mathrm{L}$. & Boraginaceae & $\mathrm{L}$ & Fever \\
\hline 31. Hyptis suaveolens (L.) Poit. & Lamiaceae & $\mathrm{L}, \mathrm{S}$ & Stomach-ache and uterus complaints \\
\hline 32. Justicia adhatoda L. & Acanthaceae & $\mathrm{L}$ & Dysentery, cold and cough \\
\hline
\end{tabular}


(Contd.)

\begin{tabular}{|c|c|c|c|c|}
\hline & $\begin{array}{l}\text { Kyllinga nemoralis (J. R. Forst \& G. } \\
\text { Forst.) Dandy }\end{array}$ & Cyperaceae & $\mathrm{R}$ & Fever \\
\hline 34. & Leucas aspera (Willd.) Link & Lamiaceae & $\mathrm{L}$ & $\begin{array}{l}\text { Chronic skin eruption and } \\
\text { rheumatism }\end{array}$ \\
\hline 35 . & Litsea glutinosa (Lour.) Roxb. & Lauraceae & $\mathrm{B}$ & Diarrhoea and dysentery \\
\hline 36. & Melia azedarach $\mathrm{L}$. & Meliaceae & $\mathrm{L}$ & Fever, small pox and antiseptic \\
\hline 37. & Mikania cordata (Burm. f.) Robin. & Asteraceae & $\mathrm{L}$ & Itches, wounds and dyspepsia \\
\hline 38. & Mimosa pudica L. & Mimosaceae & $\mathrm{R}$ & Blood diseases, jaundice and ulcer \\
\hline 39. & Moringa oleifera Lamk. & Moringaceae & $\mathrm{L}, \mathrm{Fr}, \mathrm{B}$ & $\begin{array}{l}\text { Abscesses, vomiting, dysentery, cold } \\
\text { and cough }\end{array}$ \\
\hline 40. & Nymphaea nouchali Burm. f. & Nymphaeaceae & $\mathrm{Fl}, \mathrm{Rh}$ & $\begin{array}{l}\text { Blood dysentery, piles, dyspepsia } \\
\text { and diabetes }\end{array}$ \\
\hline 41. & Ocimum tenuiflorum $\mathrm{L}$. & Lamiaceae & $\mathrm{L}$ & $\begin{array}{l}\text { Cold, cough, ring worm and gastric } \\
\text { disorders }\end{array}$ \\
\hline 42 . & Oxalis corniculata $\mathrm{L}$. & Oxalidaceae & $\mathrm{L}$ & Antiscorbutic and antidote \\
\hline 43. & Peperomia pellucida (L.) H. B. \& K. & Piperaceae & $\mathrm{L}$ & Asthma \\
\hline 44. & Phoenix sylvestris Roxb. & Arecaceae & $\mathrm{Fr}$ & $\begin{array}{l}\text { Fever, heart disease and abdominal } \\
\text { complaints }\end{array}$ \\
\hline 45 . & Phyllanthus emblica L. & Euphorbiaceae & $\mathrm{Fr}$ & Diuretic and laxative \\
\hline 46. & Piper longum L. & Piperaceae & Fr & Bronchitis, jaundice and fever \\
\hline 47. & Portulaca oleracea L. & Portulacaceae & $\mathrm{L}$ & Ulcer, diarrhoea, dysentery \\
\hline 48. & Rumex maritimus L. & Polygonaceae & $\mathrm{L}, \mathrm{S}$ & Aphrodisiac and burning complaints \\
\hline 49. & Saraca asoca (Roxb.) Willd. & Caesalpiniaceae & $\mathrm{Fl}, \mathrm{B}$ & Dysentery and female diseases \\
\hline 50. & Scoparia dulcis L. & Scrophulariaceae & WP & Diabetes and kidney problems \\
\hline 51. & Senna occidentalis Roxb. & Caesalpiniaceae & $\mathrm{L}$ & Sores and itches \\
\hline 52. & S. sophera (L.) Roxb. & Caesalpiniaceae & $\mathrm{L}$ & $\begin{array}{l}\text { Hopping cough, asthma and ring } \\
\text { worm }\end{array}$ \\
\hline 53. & Sida cordata (Burm. f.) Borss. & Malvaceae & $\mathrm{Fl}, \mathrm{Fr}$ & Burning complaints \\
\hline 54. & Solanum indicum L. & Solanaceae & $\mathrm{R}$ & Toothache and cough \\
\hline 55. & S. torvum Sw. & Solanaceae & $\mathrm{Fr}, \mathrm{R}$ & Spleen enlargement and foot crack \\
\hline 56. & Spilanthes calva DC. & Asteraceae & $\mathrm{R}$ & Toothache \\
\hline 57. & Syzygium cumini (L.) Skeels & Myrtaceae & $\mathrm{B}, \mathrm{L}, \mathrm{S}$ & $\begin{array}{l}\text { Diabetes, chronic diarrhoea and sore } \\
\text { throats }\end{array}$ \\
\hline 58. & $\begin{array}{l}\text { Terminalia arjuna (Roxb. ex DC.) } \\
\text { Wight \& Arn. }\end{array}$ & Combretaceae & $\mathrm{B}$ & Heart disease \\
\hline 59. & T. bellirica (Gaertn.) Roxb. & Combretaceae & $\mathrm{Fr}$ & $\begin{array}{l}\text { Bronchitis, diarrhoea and } \\
\text { menstruation problems }\end{array}$ \\
\hline 60. & T. chebula Retz. & Combretaceae & $\mathrm{Fr}$ & $\begin{array}{l}\text { Constipation, dysentery, } \\
\text { menstruation }\end{array}$ \\
\hline 61. & Tinospora cordifolia Miers & Menispermaceae & $\mathrm{L}, \mathrm{S}$ & $\begin{array}{l}\text { Blood purifier, jaundice and cardiac } \\
\text { problems }\end{array}$ \\
\hline 62. & Vetiveria zizanioides (L.) Nash & Poaceae & $\mathrm{R}$ & Vomiting and stomach-ache \\
\hline
\end{tabular}

$\mathrm{R}=$ Root, $\mathrm{Rh}=$ Rhizome, $\mathrm{St}=$ Stem, $\mathrm{B}=$ Bark, $\mathrm{C}=\mathrm{Corm}, \mathrm{L}=$ Leaves, $\mathrm{Fl}=$ Flower, $\mathrm{Fr}=$ Fruit, $\mathrm{S}=\mathrm{Seed}, \mathrm{WP}=$ Whole plant.

In addition to the medicinal plants used for treatment of several diseases, the inhabitants of Dhamrai employ several wild plants as leafy vegetables, e.g. Colocasia esculenta, Nymphaea nouchali, Oxalis corniculata, Solanum torvum, etc. The local people also use some plants in their religious festivals, i.e. Aegle marmelos, Ficus benghalensis, F. religiosa, Mangifera indica, Ocimum tenuiflorum, Cocos nucifera, etc. 

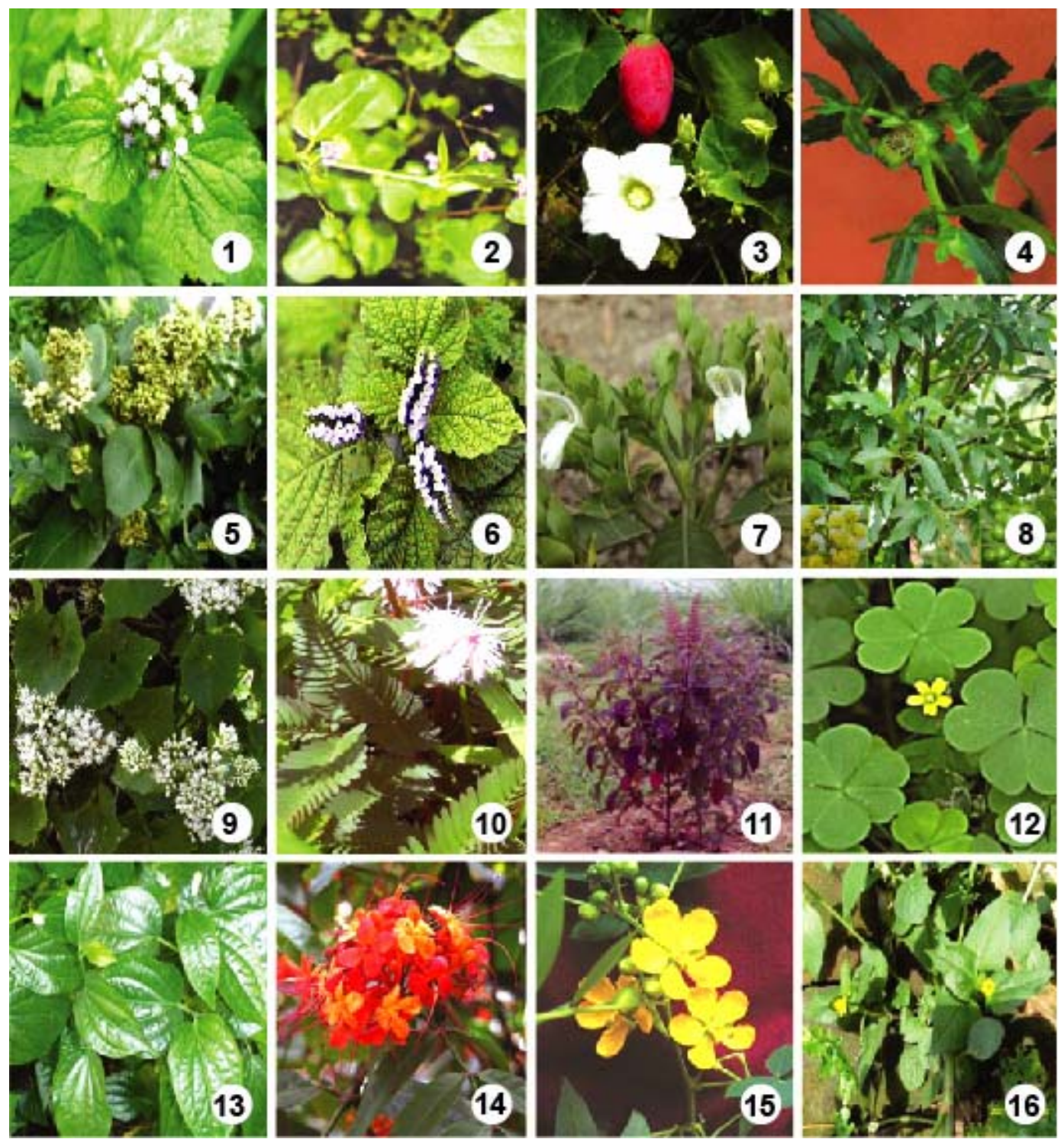

Plate 1

Figs 1-16: Some medicinal plants of Dhamrai area. 1. Ageratum conyzoides, 2. Boerhavia repens, 3. Coccinia grandis, 4. Enhydra fluctuans, 5. Glycosmis pentaphylla, 6. Heliotropium indicum, 7. Justicia adhatoda, 8. Litsea glutinosa, 9. Mikania cordata, 10. Mimosa pudica, 11. Ocimum tenuiflorum, 12. Oxalis corniculata, 13. Piper longum,14. Saraca asoca, 15. Senna occidentalis, 16. Spilanthes calva.

Out of 263 angiosperms recorded from Dhamrai area, 221 species are wild and 42 tree species are planted. Cultivated herbs and shrubs were not taken into consideration in this account. Among the 62 medicinal plants used by the local people for their primary health care 50 species have been found wild. Luxuriant growth of aquatic plants is found in Dhamrai. The macrophytes which are very common in the study area include Eichhornia crassipes, Pistia stratiotes, Aponogeton natans, 
Vallisneria spiralis, etc. Shallow water bodies support Nymphaea nouchali, Ipomoea aquatica and Enhydra fluctuans. Commonly found climbers are Asparagus racemosus and Tinospora cordiofolia. Vanda tessellata and Cuscuta reflexa were observed on the homestead trees as epiphyte and parasite, respectively. Very commonly growing roadside plants include Heliotropium indicum, Mimosa pudica, Spilanthes calva, Croton bonplandianus, Sporobolus diander, Leucus aspera and Senna tora. Several plants are found rare and endangered in Dhamrai. Some of the rare plants of Dhamrai are Alternanthera paronychioides, Amorphophallus campanulatus, Dipteracanthus prostratus, Ficus rumphii, Heliotropium ovalifolium, Hygrophila schulli, Persicaria barbata, Solanum torvum, Syngonium podophyllum and Vitex negundo.

Threats to the species and suggested measures: Plant diversity of Dhamrai is under great risk because of many threats which have been identified based on the field observations and group discussion with local people during the field visits. Some of the important threats include modern agriculture, urbanization, lack of knowledge of collection techniques particularly for medicinal plants, over-exploitation, lack of awareness, exotic plantation, etc. If the current situation goes on, the area will lost a number of important plants including the medicinal ones. Therefore, immediate steps should be undertaken to conserve the plant species along with habitat protection. In order to conserve plant diversity and to ensure habitat protection the following recommendations are suggested: (i) extensive inventory of the flora of the area should be done immediately along with distribution map of the species, (ii) conservation of medicinal and threatened plants through $e x-$ situ method should be undertaken, and the local people should be involved in this activity, (iii) awareness among the local people should be created for the conservation of botanical diversity, (iv) traditional usage of medicinal plants should be recorded and documented, (v) nursery should be developed for the propagation of threatened plants and (vi) commercial cultivation of medicinal plants should be undertaken to meet the local demand.

\section{References}

Ahmed ZU, MA Hassan, ZNT Begum, M Khondker, SMH Kabir, M Ahmad, ATA Ahmed, AKA Rahman and EU Haque (Eds) 2007-2009. Encyclopedia of Flora and Fauna of Bangladesh, Vols. 6-12. Asiatic Society of Bangladesh, Dhaka.

BBS (Bangladesh Bureau of Statistics) 2009. Monthly Statistical Bulletin, December 2009. Statistics Division, Ministry of Planning, Government of the People's Republic of Bangladesh.

Cronquist A 1981. An Integrated System of Classification of Flowering Plants. Columbia University Press, New York.

Dassanayake MD and FR Fosberg (Eds) 1980-1985. A Revised Handbook to the Flora of Ceylon, Vols. 1-5. Amerind Publishing Co. Pvt. Ltd., New Delhi.

Hossain MF, SF Elahi and M Khondaker 2003. A comparison between ecological and conventional rice farming systems in Bangladesh. Diffuse Pollution Conference Dublin, 8C Ecology 8: 57-64.

Khan MS (Ed.) 1972-1987. Flora of Bangladesh. Nos. 1-39. Bangladesh National Herbarium, BARC, Dhaka.

Khan MS and MM Rahman (Eds) 1989-2002. Flora of Bangladesh. Nos. 40-53. Bangladesh National Herbarium, Dhaka.

Khan MS and AM Huq 2001. The Vascular Flora of Chunati Wildlife Sanctuary in South Chittagong, Bangladesh. Bangladesh J. Plant Taxon. 8(1):47-64.

Khan MS, MM Rahman, AM Huq, MMK Mia and MA Hassan 1994. Assessment of Biodiversity of Teknaf Game Reserve in Bangladesh focusing on economically and ecologically important plant species. Bangladesh J. Plant Taxon. 1(1): 21-23.

Rahman MO and MA Hassan 1995. Angiospermic Flora of Bhawal National Park, Gazipur (Bangladesh). Bangladesh J. Plant Taxon. 2(1\&2): 47-79. 
Rahman MO, MZ Uddin, E Tutul, M Begum and MA Hassan 2010. Additions to the Angiospermic flora of Runctia Sal forest, Bangladesh. Bangladesh J. Plant Taxon. 17(2): 171-185.

Rashid ME and MA Rahman 2011. Updated nomenclature and taxonomic status of the plants of Bangladesh included in Hook. f., The Flora of British India: Volume-I. Bangladesh J. Plant Taxon. 18(2): 177-197.

Tutul E, MZ Uddin, MO Rahman and MA Hassan 2009. Angiospermic flora of Runctia Sal forest, Bangladesh. I. Liliopsida (Monocots). Bangladesh J. Plant Taxon. 16(1): 83-90.

Tutul E, MZ Uddin, MO Rahman and MA Hassan 2010. Angiospermic flora of Runctia Sal forest, Bangladesh. II. Magnoliopsida (Dicots). Bangladesh J. Plant Taxon. 17(1): 33-53.

Uddin MZ and MA Hassan 2010. Angiosperm diversity of Lawachara National Park (Bangladesh): A preliminary assessment. Bangladesh J. Plant Taxon. 17(1): 9-22.

Uddin SB and MA Rahman 1999. Angiospermic flora of Himchari National Park, Cox's Bazar, Bangladesh. Bangladesh J. Plant Taxon. 6(1): 31-68.

(Manuscript received on 7 February, 2012; revised on 19 April, 2012) 